\title{
Prevalence of bone mineral density loss and potential risk factors for osteopenia and osteoporosis in rheumatic patients in China: logistic regression and random forest analysis
}

\author{
Xi Zhang ${ }^{1 \#}$, Zonglin Dai ${ }^{2 \#}$, Eric H. Y. Lau ${ }^{2 \#}$, Chunping Cui ${ }^{3}$, He Lin ${ }^{4}$, Jun $\mathrm{Qi}^{1}$, Weifeng Ni ${ }^{5}$, Like Zhao ${ }^{6}$, \\ Qing Lv ${ }^{1}$, Jieruo Gu ${ }^{1}$, Zhiming Lin ${ }^{1}$ \\ ${ }^{1}$ Division of Rheumatology, Third Affiliated Hospital of Sun Yat-sen University, Guangzhou 510630, China; ${ }^{2}$ Division of Epidemiology and \\ Biostatistics, School of Public Health, University of Hong Kong, Hong Kong, China; ${ }^{3}$ Division of Neurology, Third Affiliated Hospital of Sun \\ Yat-sen University, Guangzhou 510630, China; ${ }^{4}$ Division of Rheumatology, Fujian Provincial Hospital, Fuzhou 350000, China; ${ }^{5}$ Division of \\ Rheumatology, Second Affiliated Hospital of Shantou University Medical College, Shantou 515000, China; ${ }^{6}$ Division of Rheumatology, Beijing \\ Hospital, Beijing 100000, China \\ Contributions: (I) Conception and design: X Zhang, Z Dai, EHY Lau, J Gu, Z Lin; (II) Administrative support: None; (III) Provision of study \\ materials or patients: None; (IV) Collection and assembly of data: X Zhang, Z Dai, EHY Lau; (V) Data analysis and interpretation: X Zhang, C Cui, \\ L He, J Qi, W Ni, L Zhao, Q Lv; (VI) Manuscript writing: All authors; (VII) Final approval of manuscript: All authors. \\ \#These authors contributed equally to this work. \\ Correspondence to: Zhiming Lin. Division of Rheumatology, Third Affiliated Hospital of Sun Yat-sen University, 600 Tianhe Road, Guangzhou \\ 510630, China. Email: 1zm-zj99@163.com.
}

Background: The objective of this study was to explore the prevalence of change in bone mineral density (BMD) and the potential risk factors for osteopenia and osteoporosis in rheumatic patients.

Methods: An analytical cross-sectional study design was carried out. For this study, one thousand and seven rheumatic patients were recruited and further accepted for data collection and blood and BMD tests. The potential risk factors for osteopenia and osteoporosis in rheumatic patients were further analyzed by using both logistic regression analysis and random forest (RF) analysis.

Results: $41.1 \%$ of the male patients aged 50 years or above and $50.8 \%$ of postmenopausal patients were osteoporotic in their lumbar spine. Among these patients, the prevalence of osteoporosis in the femoral neck and total hip was $19.4 \%$ and $8.9 \%$ in men, and $27.6 \%$ and $16.5 \%$ in women respectively, while more than half of the rheumatic patients had osteopenia in the femoral neck and total hip. For men younger than 50 years and premenopausal women, BMD were lower than the health population in the femoral neck $(16.5 \%$ and $18.3 \%$ respectively) and the total hip (17.4\% and $10.4 \%$ respectively). Older age, body mass index (BMI) $<18.5 \mathrm{~kg} / \mathrm{m}^{2}$, female sex and glucocorticoid use were associated with lower BMD in the lumbar spine, femoral neck, and total hip of patients. In RF analysis, age was ranked as the most important factor for osteopenia in the lumbar spine, femoral neck, and total hip of patients, followed by glucocorticoid use and BMI.

Conclusions: More interventions should be given to osteopenia patients because of the higher prevalence when compared with osteoporosis patients. Older age, BMI $<18.5 \mathrm{~kg} / \mathrm{m}^{2}$, female sex and glucocorticoid use were associated with lower BMD in rheumatic patients. The results from the logistic regression can be supplemented by random forest analysis.

Keywords: Bone mineral density; osteopenia; osteoporosis; rheumatic diseases; logistic regression; random forest analysis

Submitted Sep 09, 2019. Accepted for publication Dec 27, 2019.

doi: $10.21037 /$ atm.2020.01.08

View this article at: http://dx.doi.org/10.21037/atm.2020.01.08 


\section{Introduction}

Rheumatic diseases invade the joints, bones, muscles, blood vessels, and related soft tissues or connective tissues (1-4). Most rheumatic diseases are inflammatory diseases (1-5). Many patients tend to use glucocorticoids to treat the disease (6). However, glucocorticoid use was proved to be potential risk factors for osteoporosis according to a previous study (7). Evidence has shown that both inflammation and glucocorticoids inhibit osteoprotegerin and activate osteoclast activity, thereby affecting formation of the bone matrix and inhibiting intestinal calcium absorption $(8,9)$. Hence, rheumatic patients are more likely to suffer from osteoporosis.

Many studies have reported on the prevalence of osteopenia and osteoporosis in rheumatic patients (10-13). However, the results are often presented in the form of one specific disease type, such as systemic lupus erythematosus (SLE) and rheumatoid arthritis (RA), instead of as a whole. A retrospective study in Spain in 2010 showed a high prevalence of osteopenia (average 36.9\%) compared to osteoporosis (average 5.4\%) among 105 female patients with SLE (12). Among African Americans in the United States in 2001, around $32.9 \%$ and $4.7 \%$ of RA patients suffered from osteopenia and osteoporosis, respectively $(10,11)$. In China, the prevalence of rheumatism has varied by city and disease type (13). In 2001, the prevalence of RA was $0.34 \%, 0.52 \%$, and $0.44 \%$ in Beijing, Shanghai, and Shenzhen, respectively. However, the prevalence of symptomatic osteoarthritis (OA) was relatively higher than RA in the same city. In 2005, the prevalence of symptomatic OA was $9.56 \%$ in Beijing and $17.5 \%$ in Shanghai. The pain varied from $11.6 \%$ to $46.4 \%$ in different parts of China (13). However, studies in China on bone mineral density (BMD) in different rheumatic diseases with large sample size is rare.

Postmenopausal women are more likely than men to suffer from osteoporosis, as reported by many studies $(11,14)$. Risk factors include age and glucocorticoid use $(7,15)$. Nevertheless, more factors need to be included to explore the association and to see whether these factors enhance the association. Levels of biological characteristics, health behaviors, and medical history (such as diabetes, hypertension, and hyperthyroidism) may play a role in the decreased BMD. Some studies have shown a positive association between calcium and vitamin $\mathrm{D}$ use and higher BMD (16-18). However, others posed a different result, that is, that calcium and vitamin D use were weakly or inversely associated (19-22). Moreover, previous studies have focused mainly on the risk factors for osteoporosis instead of osteopenia, which is the end-stage of BMD change.

Traditional logistic regression has been the main method adopted by many studies. Although logistic regression is a great process, it should be used under certain assumptions, such as independent variables. Applying a machinelearning method is common and random forest (RF) can be used for analysis without the above assumption in logistic regression (23). However, we did not find any published research that has applied both logistic regression and RF analysis together to assess comprehensive risk factors for osteopenia as a first step and to predict osteoporosis as a second step in patients with osteopenia.

The traditional treatment for rheumatism with osteoporosis bring challenges to patients and healthcare workers. The present study was conducted to explore the prevalence of BMD change among patients with rheumatism and to investigate the differences between the BMD normal patients and the osteopenia patients. Then, the differences between the osteopenia patients and the osteoporosis patients were further explored by conducting a cross-sectional survey in four hospitals in different districts in China: Third Affiliated Hospital of Sun Yat-sen University, Beijing Hospital, Fujian General Hospital, and the Shantou Second General Hospital. The principal center was the Third Affiliated Hospital, Sun Yat-sen University.

\section{Methods}

\section{Study design and population}

An analytical cross-sectional study design was carried out, and 1,200 rheumatic patients were selected considering the inclusion and exclusion criteria from the rheumatism departments in four hospitals from May 2018 to August 2018. Patients who were aged 18 or older and diagnosed with rheumatism for at least one year and who had provided written informed consent were included. Exclusion criteria included when patients were unable to answer questions, were pregnant, refused to write informed consent, or dropped out in the process of this study.

The minimum sample size was calculated based on the formula $\mathrm{n}=Z^{2} p q / d^{2}$ (where, $\mathrm{z}=$ critical value normal distribution, $\mathrm{p}=$ the expected prevalence, $\mathrm{q}=1-\mathrm{p}, \mathrm{d}=$ tolerable error of the prevalence) (24). A systematic sampling design was used to recruit the patients. We first selected the sample unit in line with the province in which the patient lived, followed by the second sample unit (city). 
The final sampling included 26 provinces and 82 cities. Considering the expulsion rate and incomplete feedback, we finally recruited 1,007 patients for this study.

\section{Data collection, procedures, and tools}

We categorized rheumatism into seven types: rheumatoid arthritis, systemic lupus erythematosus, osteoarthritis, spondyloarthritis, gout, Sjogren's syndrome, and others. A five-part BMD questionnaire was designed for this study. The first part of this questionnaire contained demographic information such as age, date of birth, gender, residence, height, weight, menopause age, etc. Part two consisted of the patient's lifestyle habits including drinking and smoking and drug use, including calcium, vitamin $\mathrm{D}$, and hormonal drugs. All of the questions in part two were dichotomous except hormonal drugs, which was a continuous variable. The third part focused on their medical history, such as diabetes mellitus type 2 and hypertension, and part four of the questionnaire consisted of a biochemical examination. The $\mathrm{T}$ - and the $\mathrm{Z}$-score of the lumbar spine, femoral neck, and total hip were recorded in the last part of the questionnaire.

The collection procedures were in two stages. The patients filled in the first part of the questionnaire after admission under the guidance of the trained researcher/ interviewers. To reduce the impact of the test report on patients, the other parts were completed by the communitylicensed physician according to the patients' rheumatism guidebook and inspection report after the patient had finished the blood test and BMD test at the hospital.

Body mass index (BMI) was calculated by dividing weight $(\mathrm{kg})$ by height $\left(\mathrm{m}^{2}\right)$. The weight and height were measured with the patient standing barefoot on an electronic scale with minimal clothes. The physician then recorded the data of weight and height. Smoking and drinking habits, medical history, and medications were taken from each patient's history. Patients were divided into current/former smokers and non-smokers. Drinking status was categorized as always or never/seldom.

\section{Blood samples and BMD tests}

Fresh blood samples were collected from each patient after the patient had been admitted. Blood calcium (BC) and serum phosphate (SP) concentrations were determined from samples collected in an EDTA tube. Concentrations were measured by an auto-analyzer (Hitachi 7600, Tokyo,
Japan). After the blood samples had been taken, the patients were taken to the nuclear medicine department for the BMD determination in the lumbar spine, femoral neck, and total hip by using bone $\mathrm{X}$-ray absorptiometry (Hologic 0101549, Bedfordshire, USA).

\section{Definitions}

The definition of the two measures, the T- and the Z-score, according to the World Health Organization (25), generates the results of BMD. Scores signify the deviation from the mean in terms of one's BMD and a lower score indicates a lower BMD. A T-score of -1.0 or above, from -1.0 to -2.5 , and -2.5 or lower represent the normal condition, osteopenia, and osteoporosis, respectively. All of them can be seen as three sequential stages. Meanwhile, the $\mathrm{Z}$-score indicates the difference between the BMD of the measured person with healthy people of the same gender and age. A Z-score of -2.0 or lower means a lower BMD compared to the peers. Meanwhile, according to the definition by WHO, BMI is categorized as malnutrition, normal, and overweight when the individual had a BMI of $<18.5, \geq 18.5$ to $<25$, and $\geq 25$ to $<30$, respectively. In our study, a patient with a BMI over 30 was considered obese.

\section{Data processing and statistical analysis}

Data were entered into Microsoft Office Excel (version 2016), and then two of the physicians rechecked and transferred this data to the $\mathrm{R}$ software (version 3.6.1) for analysis. Continuous variables were marked as the mean \pm standard error while discontinuous variables were presented as frequency and percentage. Independent variables consisted of age, gender, BMI, medical history, smoking, and drinking. Dependent variables were the T-score of BMD of the lumbar spine, femoral neck, and total hip, respectively, and each of the variables had been transferred into polytomous variables including normal, osteopenia, and osteoporosis. However, a T-score of BMD was suitable for men over 50 and postmenopausal women. The Kruskal-Wallis test was used for the distribution of rheumatism based on patient age. Pearson's Chi-squared test or Fisher's exact test was used, with simulated P value based on 2,000 replicates for zero counts. A P value less than 0.05 was considered statistically significant. RF was based on an ensemble of classification trees in which it split the data into several nodes that maximized the homogeneity in each group, the random forest assembled hundreds more 
classification trees with a selection of correlates randomly. The Gini index represented the purity of the nodes and it used as a reference the relative importance of the correlates. We first examined the association between these factors and osteopenia among patients with normal BMD or osteopenia using logistic regression (LG1) and random forest (RF1) analysis, then associations with osteoporosis among patients with osteopenia or osteoporosis (LG2 and RF2). Adjusted odds ratios (aORs) with 95\% confidence interval (CI) were presented in the logistic regression analysis. To assess the performance of logistic regression and RF, we randomly split the sample of rheumatic patients into training set and validation set at a ratio of 1:1. The validation set was used to test the accuracy of the model built by the training set. In each model, the bag size was equivalent to the sample size of the training set corresponding to the specific analysis, and sampling in each bag was carried out without replacement. The total number of trees grown was 500 and the minimum number of instance per leaf was 1 . All available predictor variables (11 features) were included in the random forest. We present the out-of-bag (OOB) error rate for RF1 and RF2 and the test error rate on the validation sets for all models.

\section{Results}

\section{Baseline characteristics of patients}

A total of 1,007 patients participated in this study. The basic demographic characteristics of the patients are presented in Table 1. Men older than 50 years and postmenopausal women totaled $690(68.5 \%)$ patients. In this study, rheumatoid arthritis was the most common disease (32.8\%) of the rheumatic diseases followed by systemic lupus erythematosus (13.3\%) and osteoarthritis (10.9\%). The distribution of BMD among patients is shown in Tables 2,3.

\section{Prevalence of BMD change}

As shown in Tables 2, around half of the men older than 50 years $(41.1 \%)$ and postmenopausal women $(50.8 \%)$ had osteoporosis in their lumbar spine. Although the prevalence of osteoporosis in the femoral neck and total hip was $19.4 \%$ and $8.9 \%$ in men and $27.6 \%$ and $16.5 \%$ in women respectively in this group, the prevalence of osteopenia exceeded $50 \%$ in the femoral neck and total hip for both men and women. Moreover, the prevalence among patients of normal BMD in the lumbar spine $(13.9 \%$ for men and
$13.1 \%$ for women) and femoral neck (19.4\% for men and $16.7 \%$ for women) was relatively lower than that in total hips.

For men younger than 50 years, $47.8 \%, 16.5 \%$, and $17.4 \%$ of patients had a BMD lower than the aged-matched rheumatic patients in the lumbar spine, femoral neck and total hip, while the fractions were $20.3 \%, 18.3 \%$ and $10.4 \%$ respectively for premenopausal female (Table 3). For stratification by gender and BMI when considering the T-score, osteopenia was more prevalent than osteoporosis, but both accounted for the major proportion of lower BMD. On the contrary, in the $\mathrm{Z}$-score when the BMD of patients was compared to the patients' peers, the normal group took up the main proportion. However, in stratification, men showed a similar percentage in the lumbar spine and the malnourished group in the lumbar spine and femoral neck.

Figure 1 shows the distribution of the rheumatic patients according to their age based on the $\mathrm{T}$-score and $\mathrm{Z}$-score in the lumbar spine, femoral neck, and total hip. When considering the T-score, the median ages of women who had osteopenia in the lumbar spine, femoral neck, and total hip were 55,60, and 61 years respectively, which were lower than men. However, in terms of total hip, women with osteoporosis showed a higher median age (65 years old) than men (60 years old) with osteoporosis. It was not statistically significant between men and women when their $Z$-score values were less than -2 in the femoral neck and total hip.

\section{Odds ratio and RF analysis for osteopenia and osteoporosis}

Table 4 shows the relationships among the criteria of the BMD and variables in men older than 50 years and postmenopausal women using a logistic-adjusted regression model in the lumbar spine, femoral neck, and total hip. The corresponding results of three models of osteopenia in LG1 showed that the area under curve (AUC) values were $65.4 \%, 67.5 \%$, and $70.4 \%$ respectively, whereas for osteoporosis in LG2, the AUC values were $63.7 \%$, 67.3\%, and $70.0 \%$ respectively (Figure $S 1$ ).

In RF1 analysis, age was ranked as the most important factor for osteopenia in a patient's lumbar spine, femoral neck, and total hip (Figure 2). Given the Gini index $>5$, the important factors were mostly the same as the logistic regression models. However, we found that hormone use was an important factor in all three RF models but was only statistically significant by the logistic regression analysis in the femoral neck. Estimated error rates (OOB 
Table 1 Demographic characteristics of the sample

\begin{tabular}{|c|c|c|c|c|}
\hline Characteristic & All patients $(\mathrm{N}=1,007), \mathrm{n}(\%)$ & Male (N=295), n (\%) & Female (N=712), n (\%) & $P$ values ${ }^{\dagger}$ \\
\hline$\geq 50$ & $636(63.2)$ & $180(17.9)$ & $456(45.3)$ & \\
\hline$<50$ & $371(36.8)$ & $115(11.4)$ & $256(25.4)$ & \\
\hline Menopause & $510(50.6)$ & - & $510(71.6)$ & \\
\hline Rheumatoid arthritis & $330(32.8)$ & $72(24.4)$ & $258(36.2)$ & \\
\hline Systemic lupus erythematosus & $134(13.3)$ & $16(5.4)$ & $118(16.6)$ & \\
\hline Osteoarthritis & $110(10.9)$ & $20(6.8)$ & $90(12.6)$ & \\
\hline Spondyloarthritis & $71(7.1)$ & $53(18.0)$ & $18(2.5)$ & \\
\hline Others & $248(24.6)$ & $78(26.4)$ & $170(23.9)$ & \\
\hline \multicolumn{5}{|l|}{ Medication } \\
\hline Hormonal drugs & $489(48.6)$ & $123(41.7)$ & $366(51.4)$ & 0.006 \\
\hline Calcium intake & $860(85.4)$ & $238(80.7)$ & $622(87.4)$ & 0.008 \\
\hline Vitamin D & $846(84.0)$ & $230(78.0)$ & $616(86.5)$ & $<0.001$ \\
\hline \multicolumn{5}{|l|}{ Medical history } \\
\hline Diabetes & $90(8.9)$ & $35(11.9)$ & $55(7.7)$ & 0.048 \\
\hline Hypertension & $221(21.9)$ & $73(24.7)$ & $148(20.8)$ & 0.190 \\
\hline $18.5-24.9$ & $644(64.0)$ & $191(64.7)$ & $453(63.6)$ & \\
\hline $25.0-29.9$ & $203(20.2)$ & $61(20.7)$ & $142(19.9)$ & \\
\hline$>30.0$ & $26(2.6)$ & $8(2.7)$ & $18(2.5)$ & \\
\hline
\end{tabular}

${ }^{\dagger}$, Pearson's Chi-squared test or Fisher's exact test. BMI, body mass index.

error estimate) for RF1 analysis were $29.2 \%, 26.1 \%$, and $34.9 \%$ for the lumbar spine, femoral neck, and total hip, respectively (Table 5).

In RF2 analysis for osteoporosis, age was still ranked as the most important factor in the three areas (Figure 3). The second important factor in the three RF models was hormone use. However, in the three logistic regression models, hormone use was not statistically significant.
Sex became the $4^{\text {th }}$ most important factor in predicting osteoporosis in lumbar spine and total hip in RF2. Compared to logistic regression, RF2 analysis mined more factors on osteoporosis in the lumbar spine. Estimated error rates (OOB error estimate) for RF2 analysis were $42.5 \%$, $34.0 \%$, and $22.4 \%$ for the lumbar spine, femoral neck, and total hip, respectively (Table 5).

If age alone or together with other important factors 


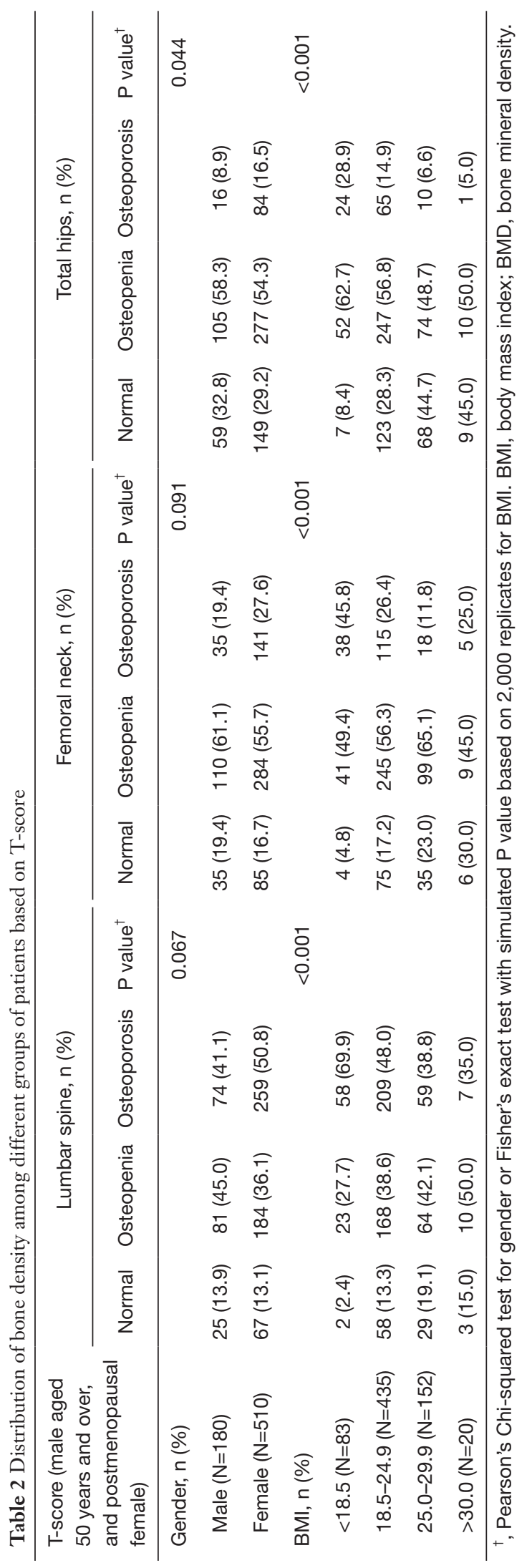

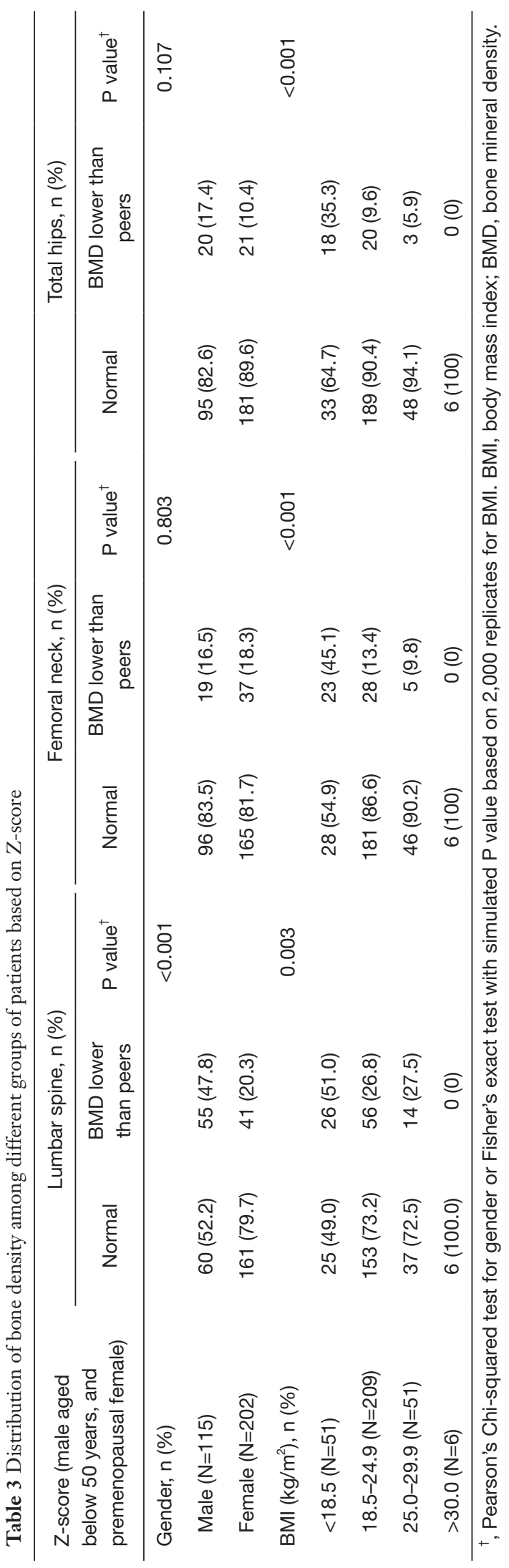



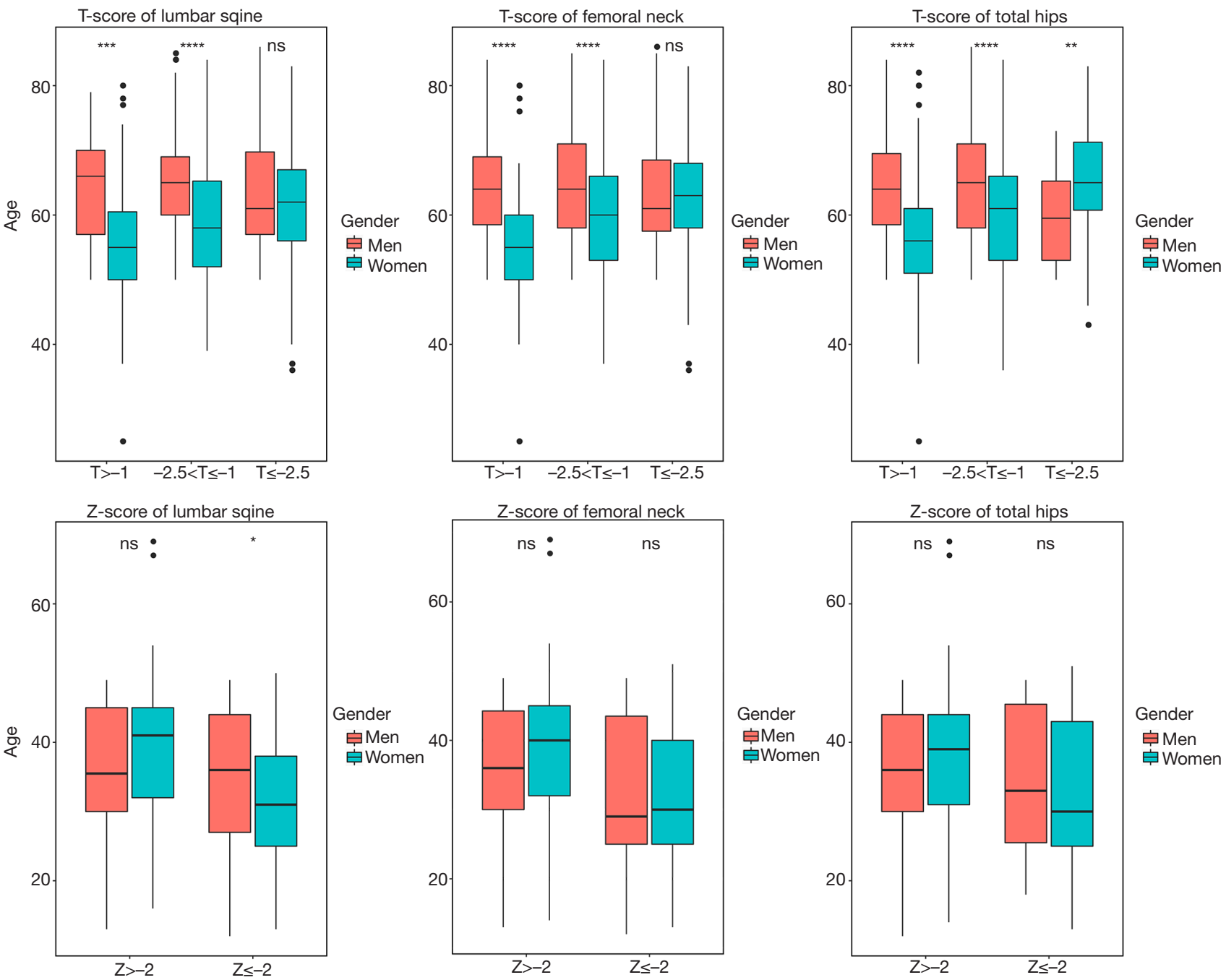

Figure 1 Distribution of rheumatic patients according to their age based on a T-score and Z-score in the lumbar spine, femoral neck, and total hip. *, $\mathrm{P} \leq 0.05 ;{ }^{* *}, \mathrm{P} \leq 0.01 ;{ }^{* * *}, \mathrm{P} \leq 0.001 ;{ }^{* * * *}, \mathrm{P} \leq 0.0001$. ns, non-significant.

was entered into the model for analysis, the OOB error estimates and the specificity and sensitivity were not improved or even attenuated (data not shown).

Sensitivity and specificity are the two main indexes to measure the prediction of the model. In Table 5, both LG1 and RF1 presented a similar performance in femoral neck and total hips. In lumbar spine, RF2 presented higher accuracy than LG2 to explore the risk for osteopenia but for osteoporosis, LG1 showed lower test error than RF1.

\section{Discussion}

The main goal of the bone mineral density investigation was to explore the prevalence of BMD change through proper stratification and improve patient and physician awareness. Such awareness could help prevent osteopenia and efficiently treat rheumatic complications of osteoporosis, and thereby enhance the quality of life for patients. There are few studies regarding the three classifications of T-score and two classifications of $Z$-score among rheumatic patients in China. Compared to previous studies that focused on the final symptom (osteoporosis) directly $(26,27)$, we picked up osteopenia as a major index and threshold. We studied the risk factors that are associated with osteopenia by using traditional logistic regression first. Because of the limitation of the logistic regression analysis not handling the multiple interactions, we analyzed the correlates by random forest analysis in order to balance the sensitivity and specificity, 
Table 4 Associations between risk factors and bone density in men older than 50 years and postmenopausal women

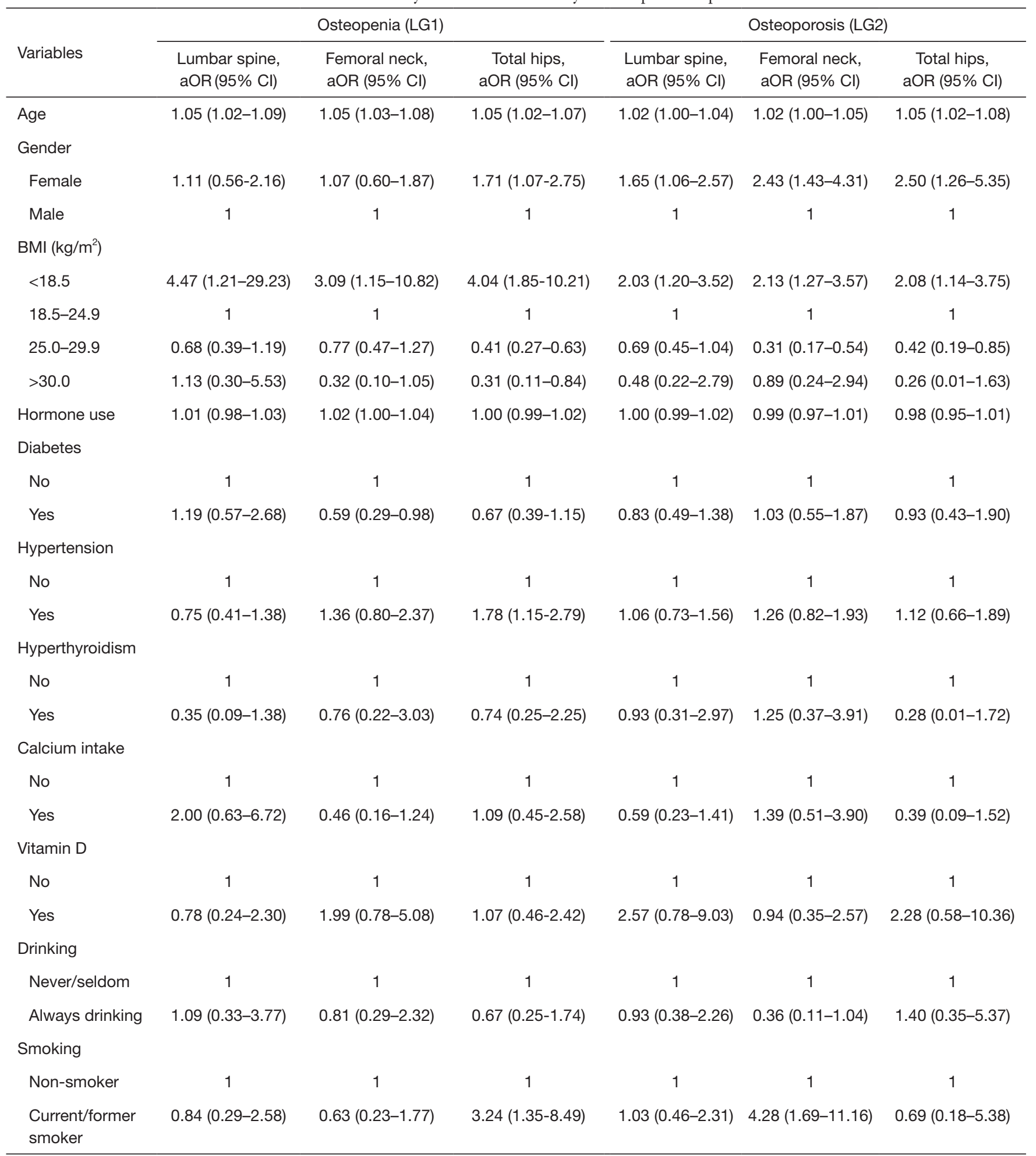

aOR, adjusted odds ratio; $\mathrm{Cl}$, confidence interval; BMI, body mass index. 


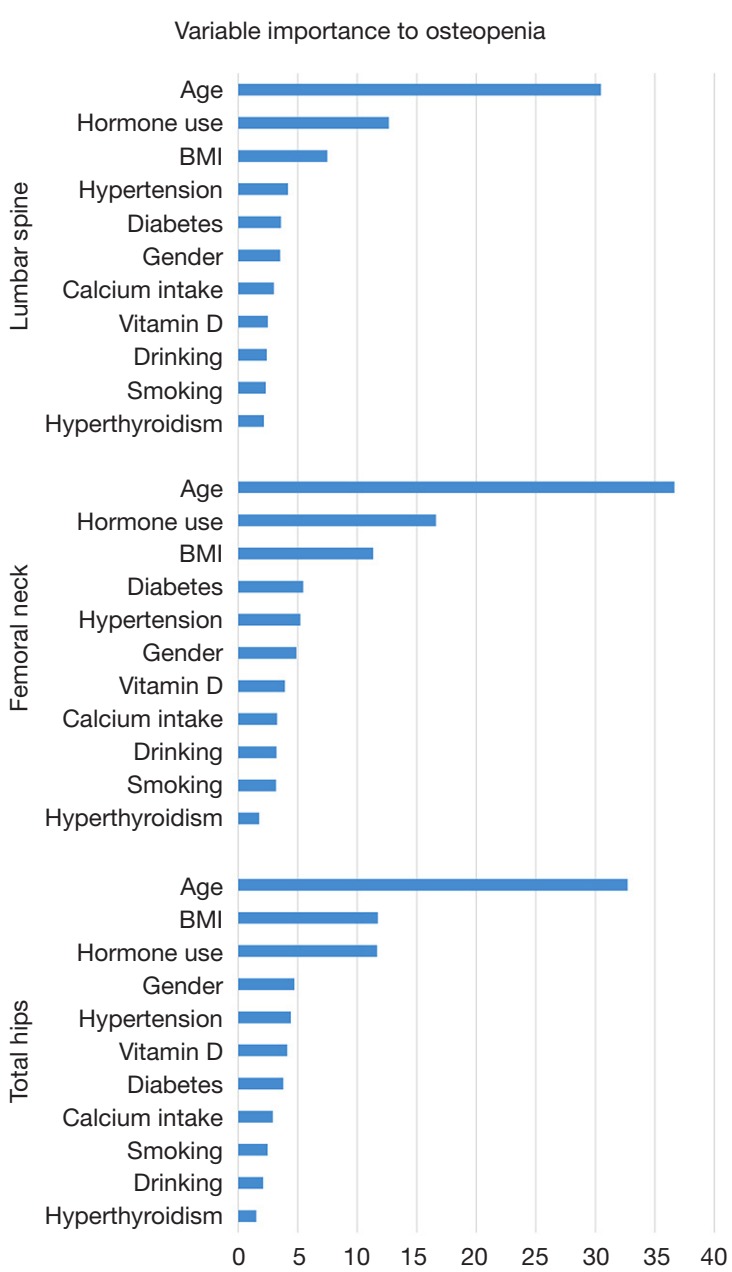

Figure 2 Permutation variable importance for osteopenia measured by the Gini index of the T-score in the lumbar spine, femoral neck, and total hip in RF1.

maximize the prediction, and reduce the confounders induced by the time interval in the development of osteoporosis. We then did the same in order to explore the risk factors for osteoporosis.

Generally, the prevalence of osteopenia was high, especially in the femoral neck and total hip of patients, and was consistent with the previous study carried out in Spain (12). The distribution of the Z-score was compared to the agematched normal group. The normal group accounted for a large proportion in the lumbar spine, the femoral neck and total hip. The reasons for the differences may be due to the statistically significant variable (age), which could result in lower BMD and was also a threshold for men older than 50 and women who had reached menopause or not. Regularly, we found that in logistic regression, the odds ratios were very close in the three anatomic locations as the patients aged and progressed from normal BMD to osteopenia and then to osteoporosis.

In the present study, we found that malnourishment affected a patient's BMD. Those with the lowest BMI were more likely to have osteopenia in the three parts of their bodies. In RF analysis, BMI was also found to be a strong predictor of osteoporosis when the patient's BMD was lower than normal. In logistic regression, a BMI $<18.5$ was associated with osteopenia and osteoporosis. However, those who were overweight but not obese tended not to have osteopenia and osteoporosis in the total hip. In addition, smoking is a risk factor for osteopenia and osteoporosis in the total hip. The possible mechanism for the impact may lie in the nicotine, which is the most abundant and most toxic substance in tobacco. Long-term exposure to nicotine can increase heart rate and blood pressure (28), lower appetite, and, most importantly, it can change the permeability of the blood vessel wall, which hinders the absorption of nutrients (29). Although we did not find an association with osteopenia in the femoral neck, patients who were current or former smokers might be more likely to have osteoporosis later, according to logistic regression. This was the case in the RF analysis in which the importance increased from the $10^{\text {th }}$ rank to the $6^{\text {th }}$ rank. What is more, patients who were current or former smokers were more likely to have osteoporosis in their condition of osteopenia and the importance level increased in RF analysis.

We also found that females were more likely than males to have osteoporosis when they had osteopenia in three parts of their bodies, according to logistic regression analysis. However, for the development of osteopenia from the normal, the result was statistically significant only in the total hip. The outcome matched the enhanced importance level and increased the Gini index in the RF analysis. What is more, according to the distribution of BMD of the Z-score stratified by age, we found that women were younger than men when they had lower BMD compared to their peers.

Hormone use played a key role in osteopenia in the femoral neck according to logistic regression analysis. However, it was an important factor in osteopenia and especially in osteoporosis in RF analysis. Apart from that, hypertension may be considered as another factor leading to osteopenia in the total hip. However, for RF analysis, the importance of hypertension became steady in the development of osteopenia and osteoporosis at the three 


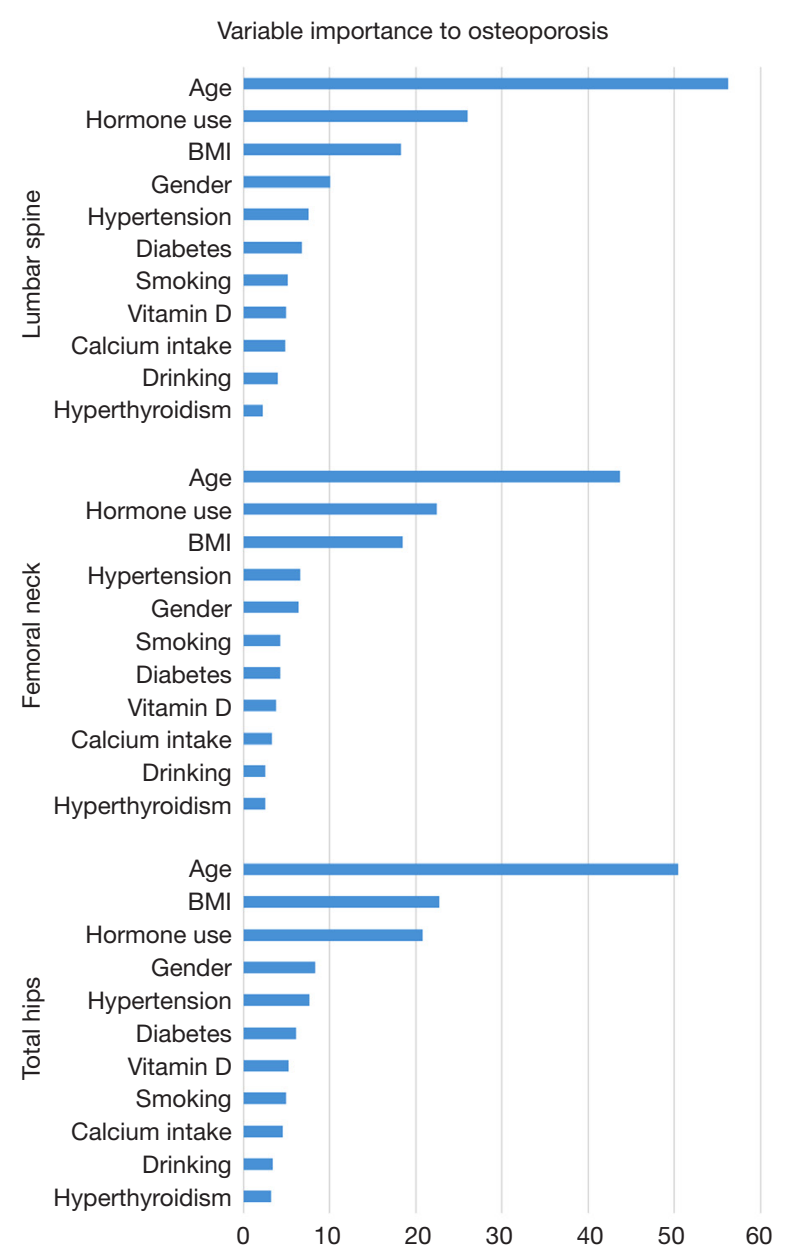

Figure 3 Permutation variable importance for osteoporosis measured by the Gini index of the T-score in the lumbar spine, femoral neck, and total hip in RF2.

anatomic sites.

Controversy exists about the association between diabetes mellitus type 2 and BMD. Our study showed patients with type 2 diabetes had an increased BMD in the femoral neck, which is consistent with previous studies; however, development of osteoporosis based on the RF analysis was not statistically significant (30). A Hertfordshire cohort study provided evidence that due to insulin resistance in the body, a large amount of insulin acted on osteoblasts through the receptor. The process was beneficial for bone formation and bone density increase (31).

Overall, the results from the logistic regression can be supplemented and strengthened by random forest analysis. The mechanism for the development of osteopenia was applied to osteoporosis. If patients were exposed to risk factors such as smoking and hormone use in the long term, they would be more likely to develop osteoporosis when they had osteopenia. Consistent with some previous studies, we found that calcium intake and vitamin D use were not protective, and the effect of vitamin $\mathrm{D}$ became attenuated for those patients in the status of osteopenia.

The strength of the study is the combination of logistic regression and random forest analysis. Besides the matched outcome in our study, the random forest analysis explored and found the interaction between correlates to be independent. For instance, the effect of hormone use may depend on the degree of the rheumatic disease, which should be included in the logistic regression. Limitations include the cross-sectional nature. In addition, the $\mathrm{BMD}$ and the diagnosis of osteoporosis relied on X-ray absorptiometry, the results of which predict fracture risk but have certain drawbacks. For example, it does not fully reflect the decline in bone mass.

\section{Conclusions}

In summary, the prevalence of osteopenia was found to be high, and it is necessary for physicians to give more intervention to those patients before osteopenia develops into osteoporosis. A combination of logistic regression and random forest model was more accurate to some extent in predicting the outcome. Older age, BMI $<18.5 \mathrm{~kg} / \mathrm{m}^{2}$, female sex and glucocorticoid use were associated with a lower BMD in the lumbar spine, femoral neck, and total hip of the patients. Each site had its own specific factors associated with osteopenia. The common potential risk factors in the lumbar spine, femoral neck, and total hip may be a pragmatic way of expanding shared efforts for the prevention of osteopenia and osteoporosis.

\section{Acknowledgments}

We thank all patients involved in the study.

Funding: This work was supported by funding from Guangdong Natural Science Funds for Distinguished Young Scholar (Grant No. 2014A030306039), High-level personnel of special support program for Technology Innovative Talents and the Top Young of Guangdong Province (Grant No. 2015TQ01R516), Distinguished Young Scholar Candidates Programme for The Third Affiliated Hospital of Sun Yat-sen University and Pearl River Nova Program of Guangzhou (Grant No. 201610010005). 


\section{Footnote}

Conflicts of Interest: The authors have no conflicts of interest to declare.

Ethical Statement: The authors are accountable for all aspects of the work in ensuring that questions related to the accuracy or integrity of any part of the work are appropriately investigated and resolved. The ethical approval was obtained from the Ethic Committee of the Third Affiliated Hospital of Sun Yat-sen University (Guangzhou, China). Informed consent was obtained from all individuals participating in this study.

Open Access Statement: This is an Open Access article distributed in accordance with the Creative Commons Attribution-NonCommercial-NoDerivs 4.0 International License (CC BY-NC-ND 4.0), which permits the noncommercial replication and distribution of the article with the strict proviso that no changes or edits are made and the original work is properly cited (including links to both the formal publication through the relevant DOI and the license). See: https://creativecommons.org/licenses/by-nc-nd/4.0/.

\section{References}

1. Hutson MA, Dharmanand BG. Soft tissue rheumatism. Ind J Rheumatol 2014;9:S1-S2.

2. Kennedy A, Ng C, Biniecka $M$, et al. Angiogenesis and blood vessel stability in inflammatory arthritis. Arthritis Rheum 2010;62:711-21.

3. Rouster-Stevens KA, Klein-Gitelman SM. Bone health in pediatric rheumatic disease. Curr Opin Pediatr 2005;17:703-8.

4. Guo Q, Wang Y, Xu D, et al. Rheumatoid arthritis: pathological mechanisms and modern pharmacologic therapies. Bone Res 2018;6:15.

5. Crowson CS, Matteson EL, Myasoedova E, et al. The lifetime risk of adult-onset rheumatoid arthritis and other inflammatory autoimmune rheumatic diseases. Arthritis Rheum 2011;63:633-9.

6. Cutolo M. Hormone therapy in rheumatic diseases. Curr Opin Rheumatol 2010;22:257-63.

7. Villa P, Moruzzi MC, Lassandro AP, et al. Glucocorticoid therapy as a significant risk factor for osteoporosis and fractures in an Italian postmenopausal population. Gynecol Endocrinol 2013;29:678-82.

8. Hofbauer LC, Gori F, Riggs BL, et al. Stimulation of osteoprotegerin ligand and inhibition of osteoprotegerin production by glucocorticoids in human osteoblastic lineage cells: potential paracrine mechanisms of glucocorticoid-induced osteoporosis. Endocrinology 1999;140:4382.

9. Saidenberg-Kermanac'h N, Cohen-Solal M, Bessis N, et al. Role for osteoprotegerin in rheumatoid inflammation. Joint Bone Spine 2004;71:9-13.

10. Mikuls T, Saag K, Westfall A, et al. High prevalence of osteopenia/osteoporosis among African Americans with Early Rheumatoid Arthritis. Arthritis Rheum 2003;48:S299.

11. Mikuls TR, Saag KG, Curtis J, et al. Prevalence of osteoporosis and osteopenia among African Americans with early rheumatoid arthritis: the impact of ethnic-specific normative data. J Natl Med Assoc 2005;97:1155-60.

12. Monte T, Ruiz JP, Torrente-Segarra V, et al. Prevalence of osteopenia and osteoporosis in women with systemic lupus erythematosus. Ann Rheum Dis 2013;72:920-1.

13. Xiang YJ, Dai SM. Prevalence of rheumatic diseases and disability in China. Rheumatol Int 2009;29:481-90.

14. Tuzun S, Eskiyurt N, Akarirmak U, et al. Incidence of hip fracture and prevalence of osteoporosis in Turkey: the FRACTURK study. Osteoporos Int 2012;23:949-55.

15. Buckley L, Humphrey MB. Glucocorticoid-Induced Osteoporosis. N Engl J Med 2018;379:2547-56.

16. Bonjour J-P, Benoit V, Rousseau B, et al. Consumption of Vitamin D-and Calcium-Fortified Soft White Cheese Lowers the Biochemical Marker of Bone Resorption TRAP 5b in Postmenopausal Women at Moderate Risk of Osteoporosis Fracture. J Nutr 2012;142:698-703.

17. Chailurkit LO, Saetung S, Thakkinstian A, et al. Discrepant influence of vitamin D status on parathyroid hormone and bone mass after two years of calcium supplementation. Clin Endocrinol (Oxf) 2010;73:167-72.

18. Lau EM, Woo J, Lam V, et al. Milk supplementation of the diet of postmenopausal Chinese women on a low calcium intake retards bone loss. J Bone Miner Res 2001;16:1704-9.

19. Bolton-Smith C, McMurdo MET, Paterson CR, et al. Two-year randomized controlled trial of vitamin $\mathrm{K} 1$ (Phylloquinone) and vitamin D 3 plus calcium on the bone health of older women. J Bone Miner Res 2007;22:509-19.

20. Riggs BL, O' Fallon WM, Muhs J, et al. Long-Term Effects of Calcium Supplementation on Serum Parathyroid Hormone Level, Bone Turnover, and Bone Loss in Elderly Women. J Bone Miner Res 1998;13:168-74. 
21. Riis B, Thomsen K, Christiansen C. Does Calcium Supplementation Prevent Postmenopausal Bone Loss? N Engl J Med 1987;316:173-7.

22. Steffensen LH, Jørgensen L, Straume B, et al. Can vitamin D 3 supplementation prevent bone loss in persons with MS? A placebo-controlled trial. J Neurol 2011;258:1624-31.

23. Lunetta KL, Hayward LB, Segal J, et al. Screening largescale association study data: exploiting interactions using random forests. BMC Genet 2004;5:32.

24. Pourhoseingholi MA, Vahedi M, Rahimzadeh M. Sample size calculation in medical studies. Gastroenterol Hepatol Bed Bench 2013;6:14-7.

25. World Health Organization. Prevention and management of osteoporosis. World Health Organization technical report series 2003;921:1.

26. Grados F, Brazier M, Kamel S, et al. Effects on bone mineral density of calcium and vitamin D supplementation in elderly women with vitamin D deficiency. Joint Bone Spine 2003;70:203-8.

Cite this article as: Zhang X, Dai Z, Lau EHY, Cui C, Lin H, Qi J, Ni W, Zhao L, Lv Q, Gu J, Lin Z. Prevalence of bone mineral density loss and potential risk factors for osteopenia and osteoporosis in rheumatic patients in China: logistic regression and random forest analysis. Ann Transl Med 2020;8(5):226. doi: 10.21037/atm.2020.01.08
27. Woodson GC. Risk factors for osteoporosis. Curr Med Res Opin 2004;20:1681-7.

28. Donny EC, Caggiula AR, Sweitzer M, et al. Selfadministered and yoked nicotine produce robust increases in blood pressure and changes in heart rate with modest effects of behavioral contingency in rats. Pharmacol Biochem Behav 2011;99:459-67.

29. Hawkins BT, Abbruscato TJ, Egleton RD, et al. Nicotine increases in vivo blood-brain barrier permeability and alters cerebral microvascular tight junction protein distribution. Brain Res 2004;1027:48-58.

30. Abdulameer SA, Sulaiman SAS, Hassali M, et al. Osteoporosis and type 2 diabetes mellitus: what do we know, and what we can do? Patient Prefer Adherence 2012;6:435-48.

31. Dennison EM, Syddall H, Aihie Sayer A, et al. Type 2 diabetes mellitus is associated with increased axial bone density in men and women from the Hertfordshire Cohort Study: evidence for an indirect effect of insulin resistance? Diabetologia 2004;47:1963-8. 

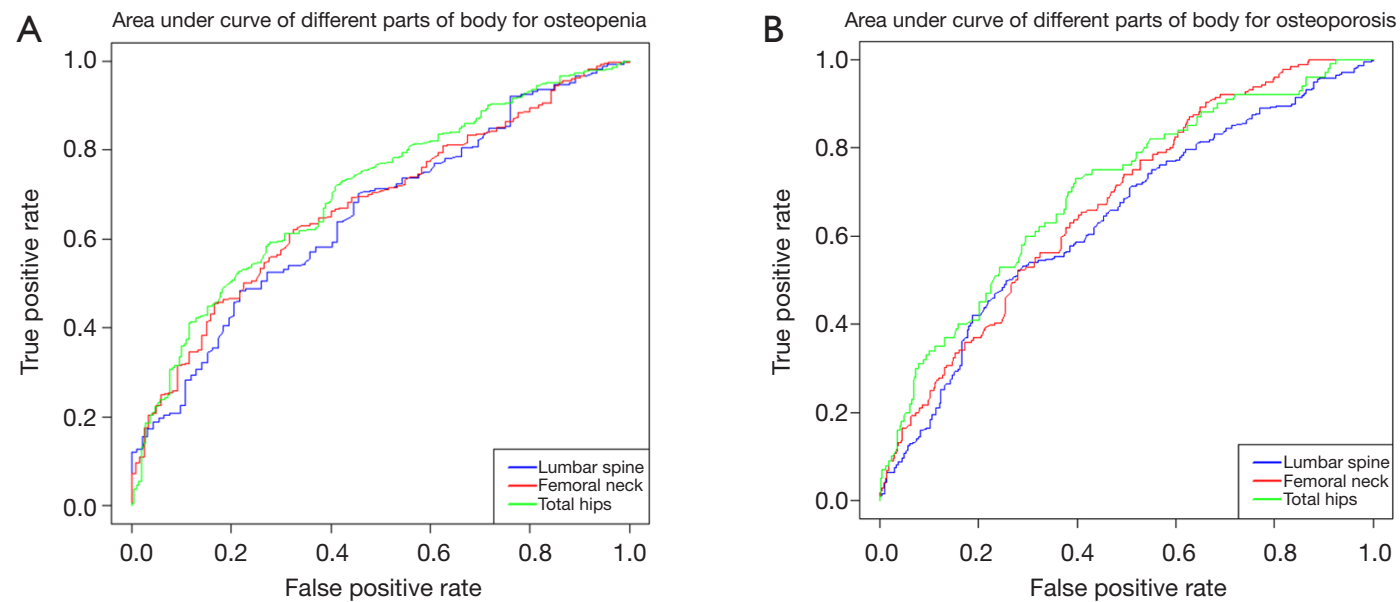

Figure S1 Area under curve (AUC) in three models of osteopenia (A) and osteoporosis (B). 